\title{
Conductive Thin Films Over Large Areas by Supramolecular Self-Assembly
}

Pei-Yang Gu, ${ }^{1,2}$ Yufeng Jiang, ${ }^{2,3}$ Zachary Fink,${ }^{4}$ Ganhua Xie, ${ }^{2}$ Qin Hu, ${ }^{2}$ Paul Y. Kim, ${ }^{2}$ Qing-Feng Xu, ${ }^{1}$ Jian-Mei Lu, ${ }^{1 *}$ and Thomas P. Russell ${ }^{2,4,5 *}$

${ }^{1}$ College of Chemistry, Chemical Engineering and Materials Science, Collaborative Innovation, Center of Suzhou Nano Science and Technology, Soochow University, Suzhou, 215123, P. R. China.

${ }^{2}$ Materials Sciences Division, Lawrence Berkeley National Laboratory, 1 Cyclotron Road, Berkeley, CA 94720, USA

${ }^{3}$ Applied Science and Technology, University of California, Berkeley, 210 Hearst Memorial Mining Building, Berkeley, CA 94720, USA

${ }^{4}$ Polymer Science and Engineering Department, University of Massachusetts, Amherst, MA 01003, USA

${ }^{5}$ Beijing Advanced Innovation Center for Soft Matter Science and Engineering, Beijing University of Chemical Technology, Beijing 100029, P. R. China. 


\section{Content}

Figure S1. AFM height images of thin films spin coated from a toluene/THF (6:4, v:v) mixture solution absent any post treatment. (a) SM1, (b) SM2, (c) SM3, (d) SM4, (e) SM5, (f) SM6, (g) SM7, (h) SM8, (i) SM9, and (j) SM10. For SM1-SM9, the $\mathrm{x}, \mathrm{y}$, and $\mathrm{z}$ scales are 1000, 1000, and $10 \mathrm{~nm}$, respectively. For SM10, the $\mathrm{x}, \mathrm{y}$, and $\mathrm{z}$ scales are 1000, 1000, and $100 \mathrm{~nm}$, respectively.

Figure S2. FFT image of AFM images of supramolecular thin films. (a) SM1, (b) SM2, (c) SM3, (d) SM4, (e) SM5, (f) SM6, (g) SM7, (h) SM8, (i) SM9, and (j) SM10, the $\mathrm{x}$ and y scales are $1000 \mathrm{~nm}$.

Figure S3. Phase contrast AFM images of thin films spin coated from a toluene/THF (6:4, v:v) mixture solution absent any post treatment. (a) SM1, (b) SM2, (c) SM3, (d) SM4, (e) SM5, (f) SM6, (g) SM7, (h) SM8, (i) SM9, and (j) SM10. For SM1-SM9, the z scale is $10^{\circ}$. For SM10, the z scale is $50^{\circ}$.

Figure S4. UV-visible absorption spectra of supramolecular thin films.

Figure S5. GIWAXS of supramolecular thin films.

Figure S6. ${ }^{1} \mathrm{H}$ NMR spectra of HOTPP in toluene- $d_{7} / \mathrm{THF}-d_{4}$.

Figure S7. ${ }^{1} \mathrm{H}$ NMR spectra of PS- $b$-P4VP (SM1), SM7, and HOTPP (SM10) in toluene- $d_{7} /$ THF- $d_{4}$.

Figure S8. GISAXS of supramolecular thin films.

Figure S9. AFM height image of SM7 thin films casted from toluene/THF (6:4, v:v) mixture solution on ITO glass without any annealing.

Figure S10. AFM height images of thin films spin coated from a toluene/THF (6:4, v:v) mixture solution absent any post treatment. (a) SML1, (b) SML2, (c) SML3, (d) SML4, (e) SML5, (f) SML6, (g) SML7, (h) SML8, and (i) SML9.

Table S1. Contact angles of the PS, P4VP, PS- $b$-P4VP, HOTPP and SM7 in toluene/THF (v:v=6:4) onto Si substrate surfaces 

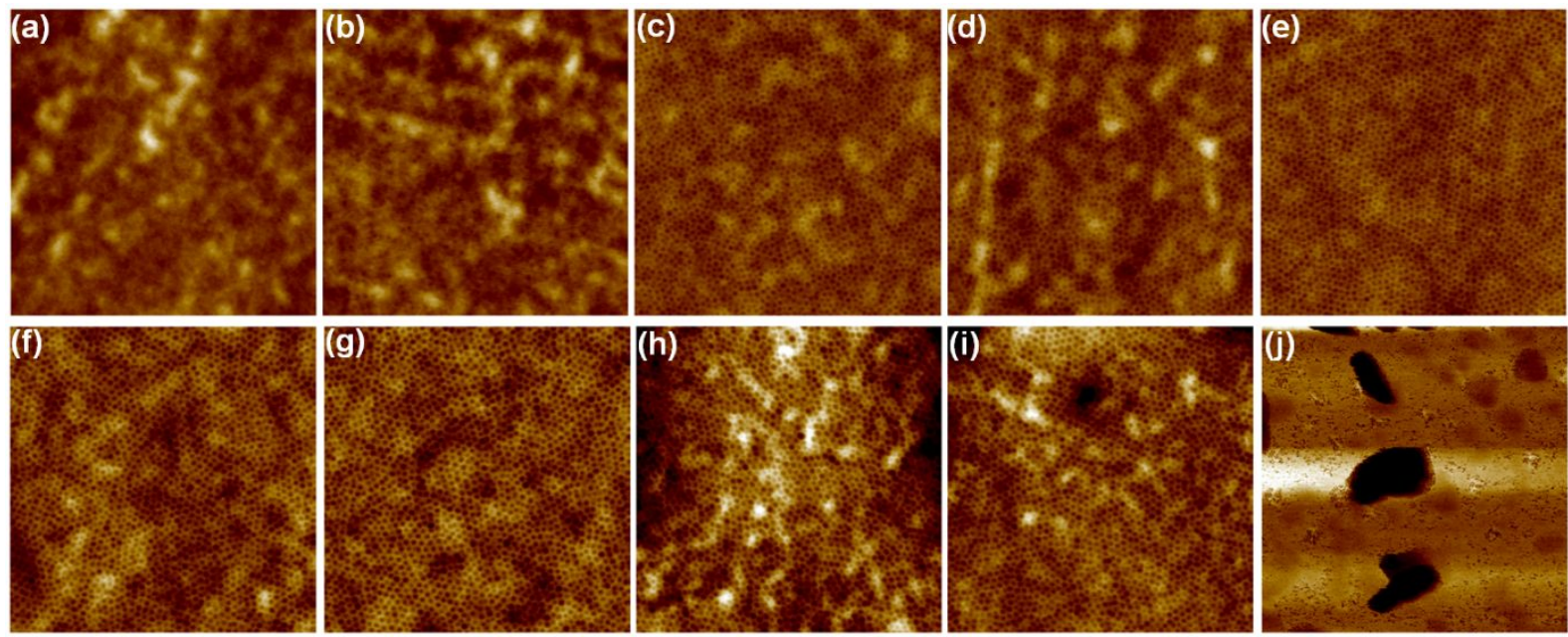

Figure S1. AFM height images of thin films spin coated from a toluene/THF (6:4, v:v) mixture solution absent any post treatment. (a) SM1, (b) SM2, (c) SM3, (d) SM4, (e) SM5, (f) SM6, (g) SM7, (h) SM8, (i) SM9, and (j) SM10. For SM1-SM9, the $\mathrm{x}, \mathrm{y}$, and $\mathrm{z}$ scales are 1000, 1000, and $10 \mathrm{~nm}$, respectively. For SM10, the $\mathrm{x}, \mathrm{y}$, and $\mathrm{z}$ scales are 1000, 1000, and $100 \mathrm{~nm}$, respectively.
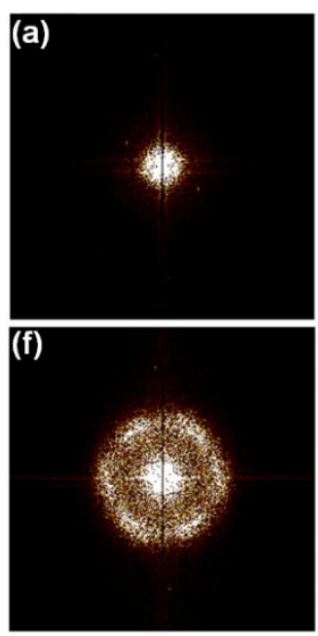
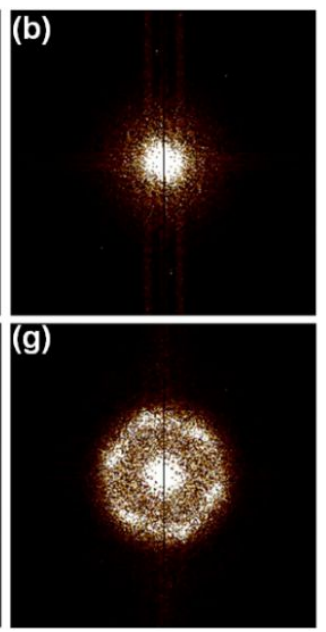
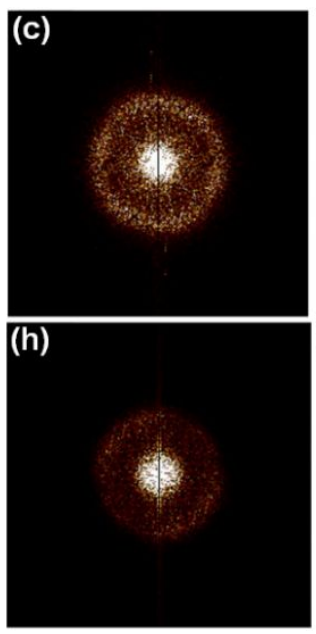
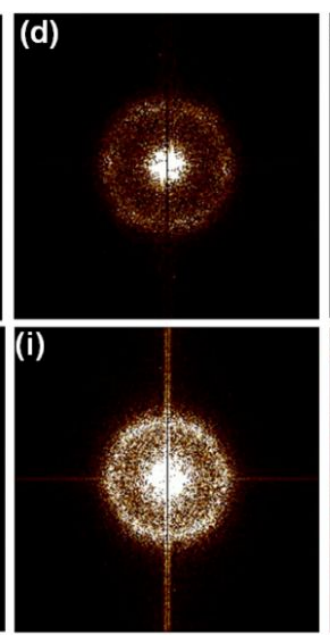
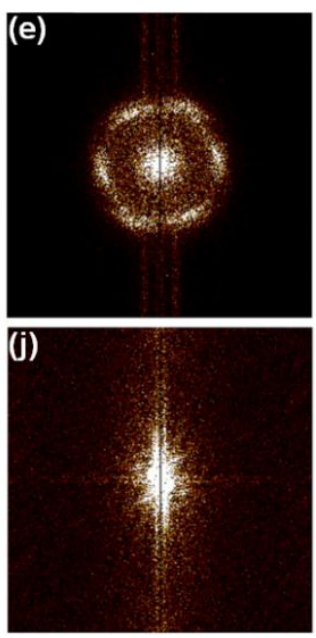

Figure S2. FFT image of AFM images of supramolecular thin films. (a) SM1, (b) SM2, (c) SM3, (d) SM4, (e) SM5, (f) SM6, (g) SM7, (h) SM8, (i) SM9, and (j) SM10, the $\mathrm{x}$ and y scales are $1000 \mathrm{~nm}$. 


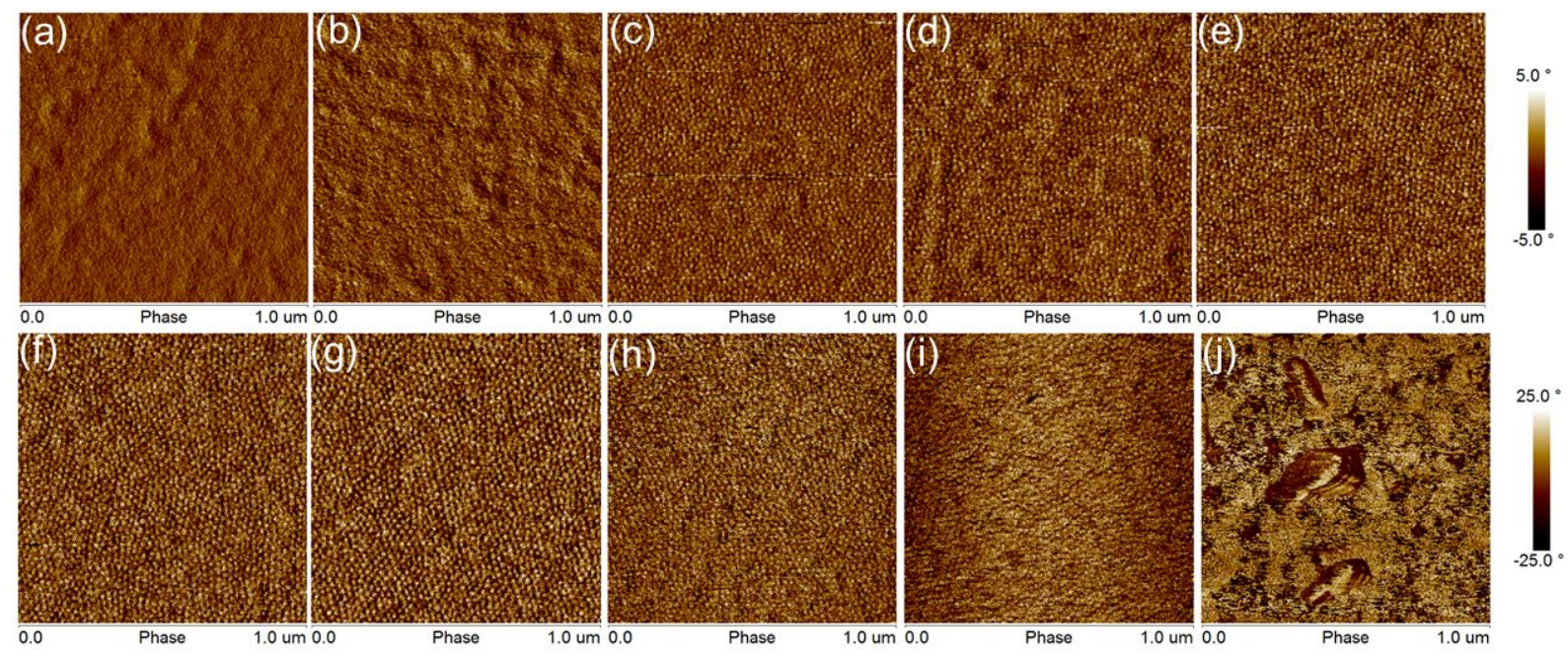

Figure S3. Phase contrast AFM images of thin films spin coated from a toluene/THF (6:4, v:v) mixture solution absent any post treatment. (a) SM1, (b) SM2, (c) SM3, (d) SM4, (e) SM5, (f) SM6, (g) SM7, (h) SM8, (i) SM9, and (j) SM10. For SM1-SM9, the $\mathrm{z}$ scale is $10^{\circ}$. For SM10, the $\mathrm{z}$ scale is $50^{\circ}$.

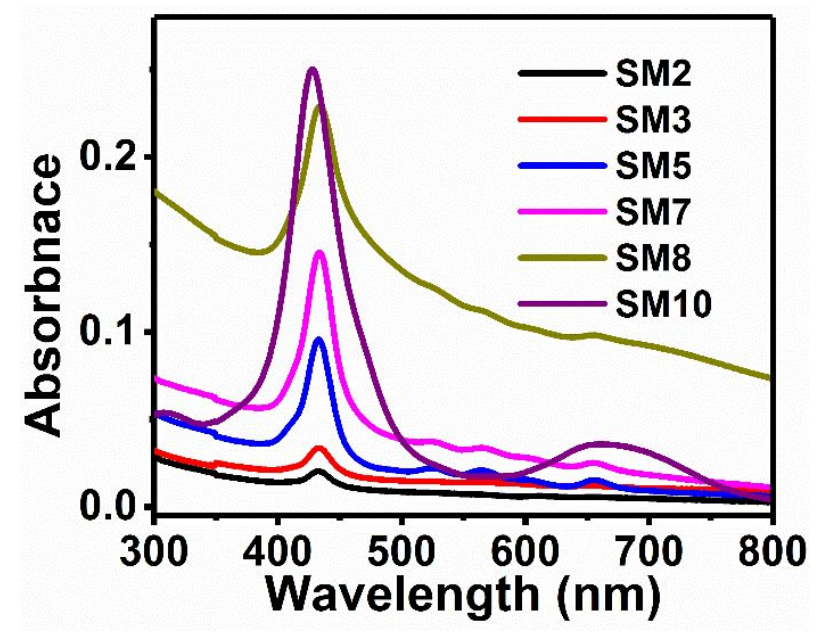

Figure S4. UV-visible absorption spectra of supramolecular thin films. 


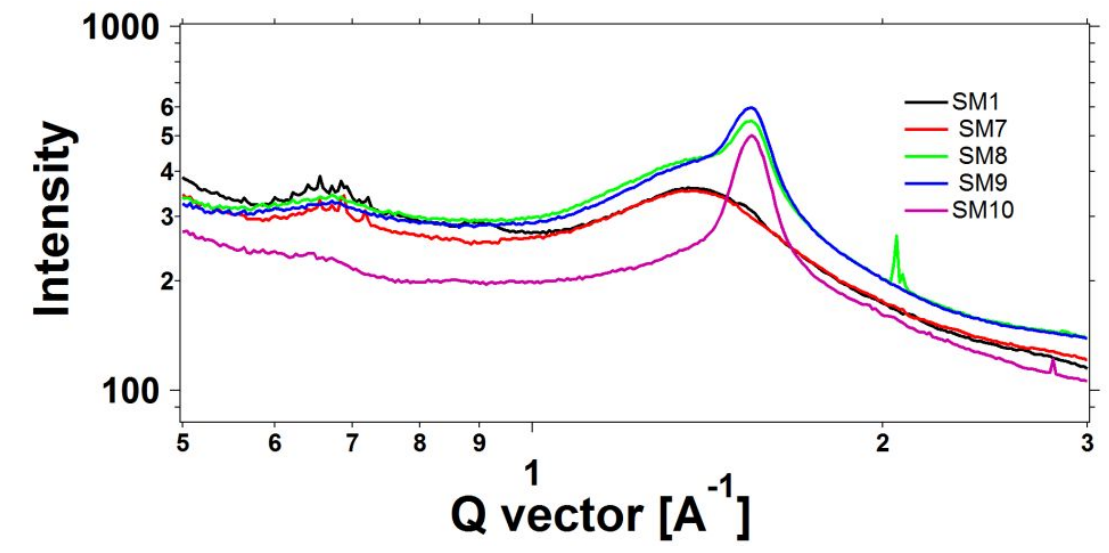

Figure S5. GIWAXS of supramolecular thin films.

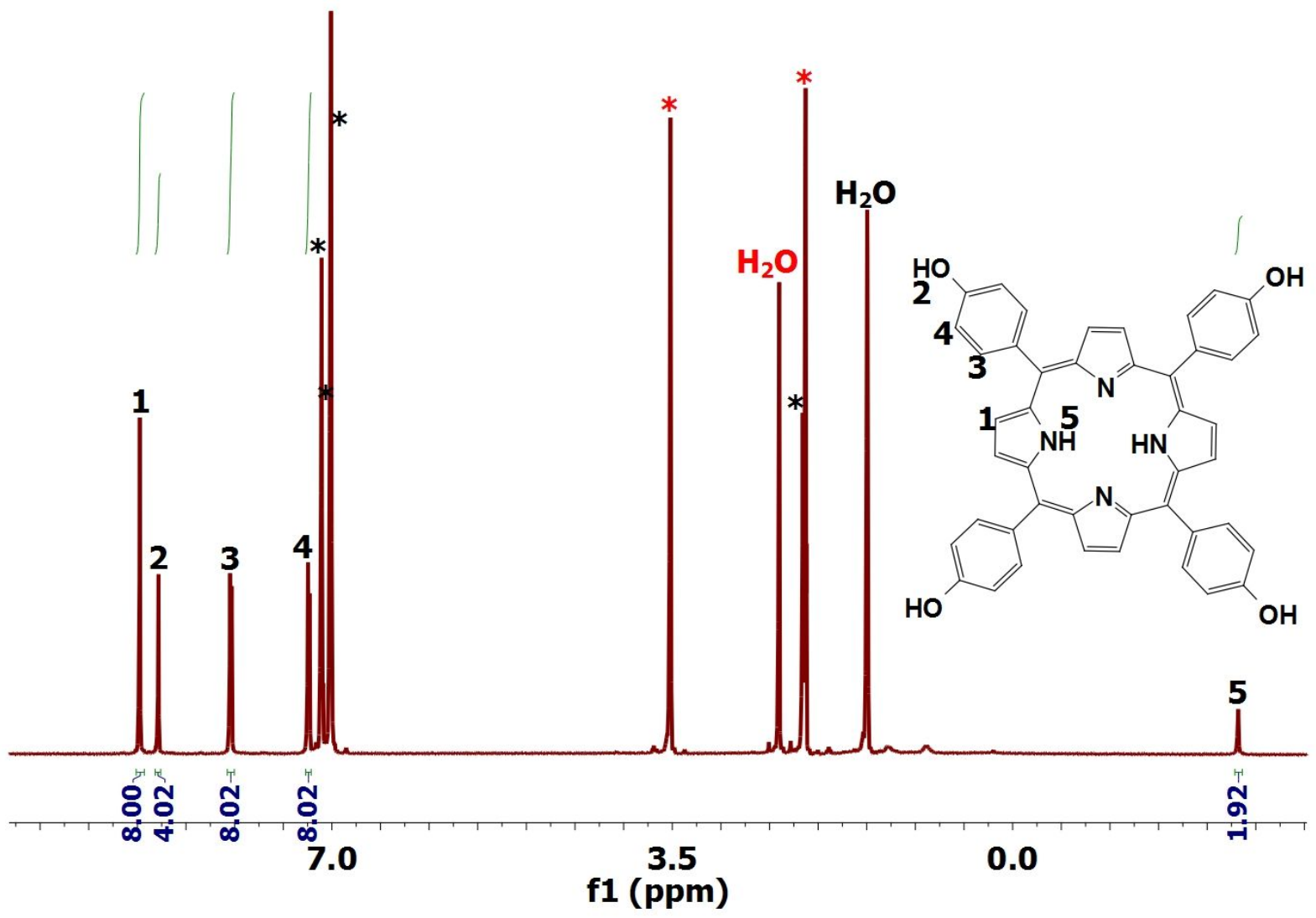

Figure S6. ${ }^{1} \mathrm{H}$ NMR spectra of HOTPP in toluene- $d_{7} /$ THF- $d_{4}$. 


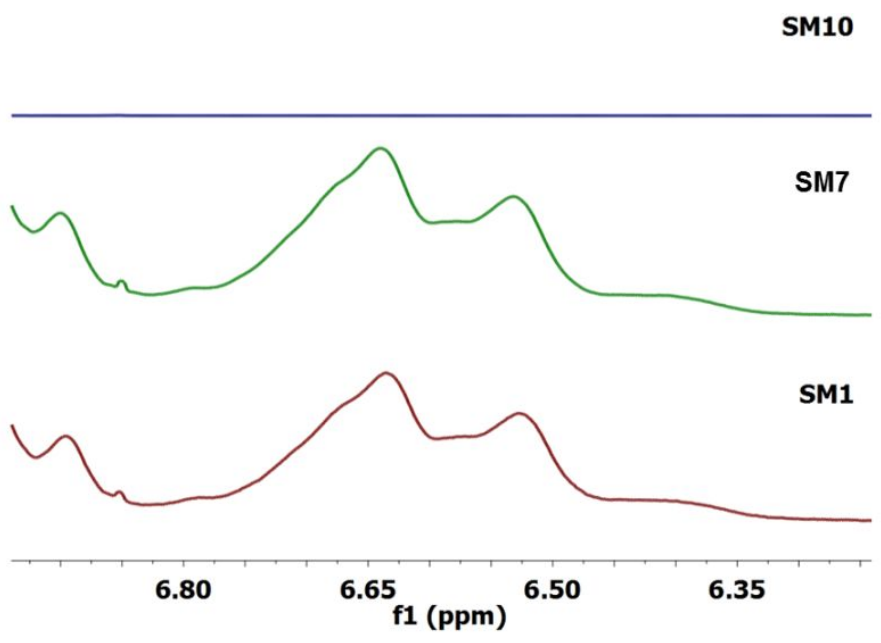

Figure S7. ${ }^{1} \mathrm{H}$ NMR spectra of PS- $b$-P4VP (SM1), SM7, and HOTPP (SM10) in toluene- $d_{7} / \mathrm{THF}-d_{4}$.

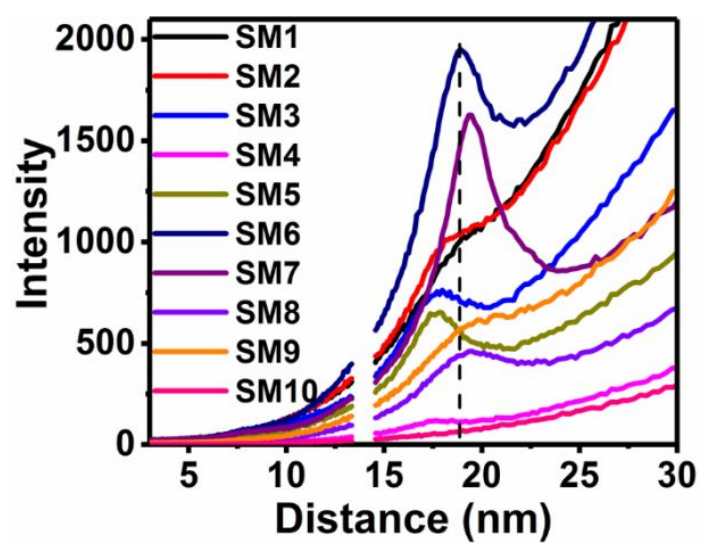

Figure S8. GISAXS of supramolecular thin films.

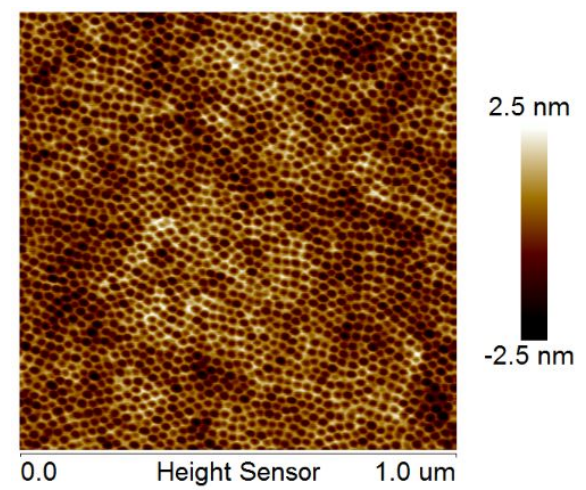

Figure S9. AFM height image of SM7 thin films casted from toluene/THF $(6: 4, \mathrm{v}: \mathrm{v})$ mixture solution on ITO glass without any annealing. 

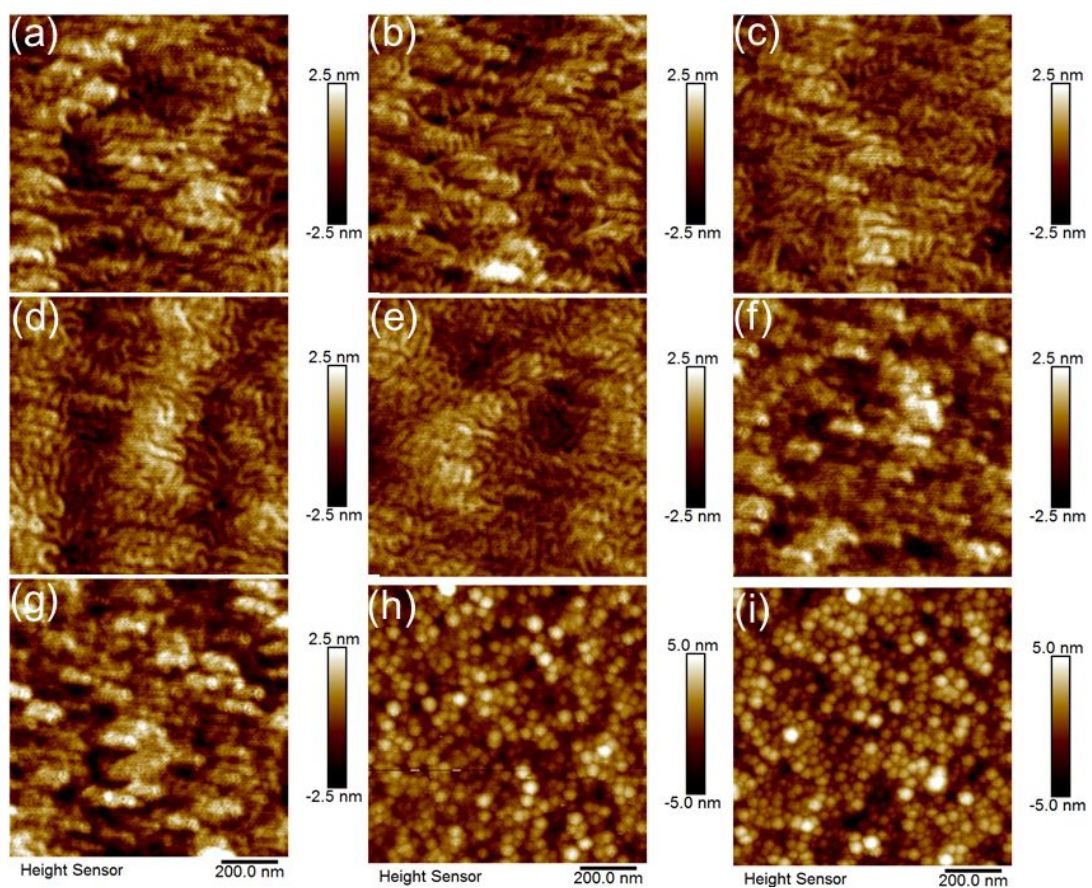

Figure S10. AFM height images of thin films spin coated from a toluene/THF (6:4, v:v) mixture solution absent any post treatment. (a) SML1, (b) SML2, (c) SML3, (d) SML4, (e) SML5, (f) SML6, (g) SML7, (h) SML8, and (i) SML9.

Table S1. Contact angles of the PS, P4VP, PS- $b$-P4VP, HOTPP and SM7 in toluene/THF (v:v=6:4) onto Si substrate surfaces

\begin{tabular}{|l|c|c|c|c|c|}
\hline Sample & PS $^{\mathbf{a}}$ & P4VP $^{\mathbf{b}}$ & PS- $b$-P4VP & HOTPP & SM7 \\
\hline Contact angle $\left({ }^{\circ}\right)^{\mathrm{c}}$ & 10.3 & 16.4 & 14.9 & 11.7 & 12.5 \\
\hline
\end{tabular}

${ }^{\mathrm{a}}$ molecular weight is $35000 \mathrm{~g}$ mol-1

${ }^{\mathrm{b}}$ molecular weight is $6000 \mathrm{~g}$ mol-1

${ }^{\mathrm{c}}$ The contact angle was measured three times per experiment, and the resulting contact angles were averaged out. 